\section{CRISP: Sleep state scoring in human infants}

\author{
STEPHEN WALKER and ROBERT EMDE \\ University of Colorado School of Medicine \\ Denver, Colorado 80200
}

In contrast to those interested in adult sleep, researchers interested in infant sleep are concerned with age-related changes in the organization of sleep states as well as with the rhythm of REM-nonREM cycles. During the newborn period, for example, organization is incomplete; the physiological and behavioral activities recorded during sleep are not readily classified into two distinct sleep states (such as REM and nonREM) because of a large amount of sleep which appears poorly organized and has mixed features. An infant sleep scoring manual was published in 1971 which resulted from an international collaborative effort to standardize terms and techniques (Anders, Emde, \& Parmclee, 1971). This manual defined three sleep states: Active REM steep, quiet sleep, and indeterminate sleep. Active REM sleep and quiet sleep were clearly distinguishable by nultiple criteria and were considered precursors of REM and nonREM sleep in the older child and adult. On the other hand, indeterminate sleep was poorly organized with mixed features and was considered an "immature" state, most characteristic of prematures and young fullterms. Indeterminate sleep was also considered characteristic of some abnormal infants.

CRISP is a computer program which automatically categorizes infant sleep using the infant sleep manual standards for coding parameters of EOG, EEG, EMG, respiration, and body movement. Multiple criteria for active REM sleep include presence of eye movements, a flat or mixed EEG, a low amplitude EMG, irregular respirations, and the presence of body movement. Multiple criteria for quiet sleep include the absence of eye movements, a high voltage or tracé alternant or mixed EEG, a high amplitude EMG, regular respirations, and the absence of body movements. The CRISP system is an adaptation and extension of Anders' RIPVAN program for use on smaller PDP-12 laboratory computers (Anders \& Zangen, 1972).

We wish to thank Dr. T. Anders for supplying the RIPVAN program and its description as well as for offering his suggestions about modifying it for the PDP-12. Dr. Emde is supported by NIMH Career Scientist Award 5 K02 MH 36808 and NIMH Grant MH 22803.
Input. Data processing begins with visual coding of each parameter for each 20-sec epoch of record. A punched paper tape is made offline. Active REM sleep and quiet sleep are then defined according to the number of parameters which satisfy the above-stated criteria. First, the program applies a system involving the least stringent criteria. When a simple majority of three out of five parameters are us d for defining either active REM or quiet sleep, all sleep will be categorized into one of these two states. Next, the critcria are made more stringent, with four out of five parameters required for either active REM or quiet sleep; this results in indeterminate sleep in addition to the original two sleep states. Finally, the criteria are made most stringent, with five out of five parameters required for assignment to one of the two sleep states, and the remaining sleep time is assigned to indeterminate sleep.

Since individual records are subject to rapid state transitions which may obscure some inherent rhythmicity in sleep state patterns, it subscquent analysis done by CRISP applies 1-min and 2 -min time smoothing to data and reruns the above analyses.

Output. Output from CRISP is in Teletype summaries and plots of both individual records and longitudinal data. Each record is analyzed in sequence until a case summary is generated which gives an overall view of a longitudinal series of records. Program subroutines carry out further analyses with outputs which automatically summarize and display the number of sleep state transitions (STABIL), the length of each state (MATUR), and the discrepant parameters in states of poor organization (DISCREP).

Computer. PDP-12, 8K, Incremental Plotter, EAE, LAP-6-DIAL.

Availability. User Manual, LINCTAPE; cost, $\$ 25.00$. Contact Stephen Walker, Department of Psychiatry, University of Colorado School of Medicine, Denver, Colorado 80220.

\section{REFERENCES}

Anders, 'T., Emde, R., \& Parmelee, A. (Eds.), $A$ manual of standardized terminology, techniques, and criteria for the scoring of states of sleep and wakefulness in newborn infants. Los Angeles: Brain Information Service, School of Health Sciences, UCLA, 1971.

Anders, T. \& Zangen, M. RIPVAN: Sleep state scoring in human infants. Psychophysiology. 1972, 9, 653-654.

(Received for publication March 28, 1975; revision received April 17, 1975.) 